\title{
Anticipatory cognitive stress appraisal modulates suppression of wound-induced macrophage activation by acute psychosocial stress
}

\author{
ULRIKE KUEBLER, ${ }^{\mathrm{a}}$ PETRA H. WIRTZ, ${ }^{\mathrm{b}, \mathrm{c}}$ MIHO SAKAI, ${ }^{\mathrm{d}}$ ANDREAS STEMMER, ${ }^{\mathrm{d}}$ REBECCA E. MEISTER, ${ }^{\mathrm{e}}$ AND \\ ULRIKE EHLERT ${ }^{a}$ \\ aDepartment of Clinical Psychology and Psychotherapy, University of Zurich, Zurich, Switzerland \\ ${ }^{\mathrm{b} B i o l o g i c a l}$ and Health Psychology, University of Bern, Bern, Switzerland \\ ${ }^{\mathrm{c} B i o l o g i c a l}$ Work and Health Psychology, University of Konstanz, Konstanz, Germany \\ ${ }^{\mathrm{d}}$ Nanotechnology Group, Swiss Federal Institute of Technology Zurich, Zurich, Switzerland \\ e Department of General Internal Medicine, Division of Psychosomatic Medicine, Inselspital, Bern University Hospital, and University of Bern, Bern, \\ Switzerland
}

\begin{abstract}
Anticipatory cognitive stress appraisal (ACSA) can affect the stress-induced release of stress hormones, which, in turn, can modulate microbicidal potential of macrophages. This study examines whether ACSA modulates wound-induced activation of macrophage microbicidal potential in 22 acutely stressed compared to 17 nonstressed healthy men. After catheter-induced wound infliction and completing the ACSA questionnaire, the stress group underwent an acute mental stress task, while the nonstressed group did not. Macrophage microbicidal potential and stress hormones were repeatedly measured. In acutely stressed men, but not in nonstressed men, higher scores in ACSA related to lower macrophage microbicidal potential. This association was statistically mediated by the norepinephrine (NE) stress response. Our data suggest that ACSA modulates stress-induced suppression of wound-induced macrophage activation and that the NE stress response underlies this effect.
\end{abstract}

Descriptors: Cognitive stress appraisal, Acute psychological stress, Macrophages, Microbicidal potential, Wound healing, Norepinephrine

Different research paradigms provide converging evidence that both acute and chronic psychological stress substantially delays skin wound healing (Gouin \& Kiecolt-Glaser, 2011; Walburn, Vedhara, Hankins, Rixon, \& Weinman, 2009). While associations between stress-induced delay of wound healing and dysregulated immune responses in the initial inflammatory phase of wound healing are well documented (Sroussi, Williams, Zhang, Villines, \& Marucha, 2009; Tymen et al., 2013; Williams, Sroussi, Abercrombie, Leung, \& Marucha, 2012), the underlying psychobiological mechanisms of this stress effect are not fully understood (Gouin \& Kiecolt-Glaser, 2011).

We recently found that wound-induced activation of microbicidal potential (i.e., superoxide anion production) of human macrophages is suppressed by acute psychosocial stress (Kuebler, Wirtz, Sakai, Stemmer, \& Ehlert, 2013). Macrophages are tissueresident immune cells that are derived from blood monocytes. During the initial inflammatory phase of wound healing, the phenotype of macrophages resembles the M1 phenotype (Daley, Brancato, Thomay, Reichner, \& Albina, 2010; Mahdavian

This work was supported by a Chocosuisse Foundation Grant 2009 (to PHW) and Swiss National Science Foundation Grant PP00P1_128565/1 (to PHW). The first two authors contributed equally to this article.

Address correspondence to: Petra H. Wirtz, Biological Work and Health Psychology, University of Konstanz, Universitaetsstrasse 10, 78457 Konstanz, Germany, E-mail: petra.wirtz@uni-konstanz.de
Delavary, van der Veer, van Egmond, Niessen, \& Beelen, 2011). M1 macrophages (also termed inflammatory macrophages) are characterized by enhanced microbicidal activity, which is mainly mediated by increased secretion of microbe-killing highly oxidizing agents, the so-called reactive oxygen species (e.g., superoxide anion, hydrogen peroxide) and the reactive nitrogen species (e.g., nitric oxide; Gordon \& Taylor, 2005). Animal studies indicate that microbicidally active M1-like macrophages play a crucial role for normal nondelayed wound healing (Lucas et al., 2010; Mori, Kondo, Nishie, Ohshima, \& Asano, 2004; Nagaoka et al., 2000; Rojas, Padgett, Sheridan, \& Marucha, 2002; Subramaniam et al., 1997). Given the importance of microbicidally active M1 macrophages for nondelayed wound healing (Mahdavian Delavary et al., 2011), our observed inhibitory effect of acute psychosocial stress on microbicidal potential of macrophages may provide a mechanism by which stress might delay skin wound healing.

While we previously found the norepinephrine (NE) stress response to modulate suppressive effects of stress on woundinduced activation of macrophage microbicidal potential, little is known about potential underlying psychological mechanisms. Previous findings from our group suggest a role for cognitive processes in modulating neuroendocrine stress responses following psychosocial stress. Anticipatory cognitive stress appraisal based on the theoretical constructs proposed by Lazarus and Folkman (1984) not only emerged as a significant determinant of stress hormone responses (Gaab, Rohleder, Nater, \& Ehlert, 2005; 
Schlotz, Hammerfald, Ehlert, \& Gaab, 2011) but also of inflammatory activity of monocytes as macrophage precursor cells following acute psychosocial stress (Wirtz, von Kanel et al., 2007). Notably, a person with higher stress appraisal is supposed to anticipate the stressor as more threatening and challenging (i.e., higher primary appraisal) and to have less competence and control in coping with the stressor (i.e., lower secondary appraisal; Gaab et al., 2005). Whether cognitive stress appraisal may similarly modulate stressinduced suppression of wound-induced macrophage activation has not yet been investigated.

The objective of the current study was to investigate in a secondary analysis of our previous study (Kuebler, Wirtz et al., 2013) whether anticipatory cognitive stress appraisal modulates the observed wound-induced activation of macrophage microbicidal potential in acutely stressed (stress wound group) compared to nonstressed men (nonstress wound group). To preactivate circulating monocytes as precursors of later M1 macrophages in vivo, we inserted a venous catheter long enough to supposedly initiate wound-healing processes (Kuebler, Ehlert et al., 2013; Kuebler, Wirtz et al., 2013). For psychosocial stress induction, we used the Trier Social Stress Test (TSST), which evokes profound psychological and physiological stress responses in a highly standardized and reliable manner (Dickerson \& Kemeny, 2004; Kirschbaum, Pirke, \& Hellhammer, 1993). M1 macrophage microbicidal potential was investigated by performing the WST-1 assay as previously described (Kuebler, Ehlert et al., 2013; Kuebler, Wirtz et al., 2013). The assay principle is based on the reduction of 2-(4-iodophenyl)3-(4-nitrophenyl)-5-(2,4-disulfophenyl)-2H-tetrazolium (WST-1) by superoxide anions produced by phorbol 12-myristate 13-acetate (PMA)-activated human monocyte-derived M1 macrophages (HMDM). As acute stress induction by TSST reliably increases anticipatory cognitive stress appraisal (Gaab et al., 2005; Het, Rohleder, Schoofs, Kirschbaum, \& Wolf, 2009; Wirtz, Elsenbruch et al., 2007), we hypothesized that stress appraisal relates to WST-1 reduction in the stress wound group. We did not expect these associations to be evident in the nonstress wound group because of too low stress appraisal scores due to the lack of stress induction. We further hypothesized that with increasing stress appraisal (i.e., increasingly higher primary appraisal in relation to secondary appraisal) stressed men would show a stronger stress-induced suppression of wound-induced activation of macrophage microbicidal potential. We also tested whether a potential association between stress appraisal and macrophage microbicidal potential would be mediated by the NE stress response.

\section{Method}

\section{Study Participants}

The current study is a secondary analysis of a study investigating effects of acute stress on wound-induced macrophage activation within a larger project (Kuebler, Wirtz et al., 2013). The Ethics Committee of the State of Zurich, Switzerland, formally approved the research protocol.

Due to known gender and menstrual cycle phase-related alterations in endocrine stress reactivity (Kirschbaum, Kudielka, Gaab, Schommer, \& Hellhammer, 1999) with potential consequences for immune responses to stress, we recruited men only. From a total of 41 participants, 39 medication-free, healthy men between 20 and 50 years of age completed the questionnaire assessing anticipatory cognitive stress appraisal. Participants were randomly assigned to either a stress wound group $(n=22)$ or a nonstress wound group $(n=17)$. Recruitment was carried out with the aid of the Swiss Red Cross of the Canton of Zurich and through advertisements. Participants were in good physical and mental health, as confirmed by a telephone interview. Explicit exclusion criteria were regular strenuous exercise, smoking, alcohol and illicit drug abuse, any heart disease, varicosis or thrombotic diseases, elevated blood sugar and diabetes, elevated cholesterol, liver and renal diseases, chronic obstructive pulmonary disease, allergies and atopic diathesis, rheumatic diseases, and current infectious diseases. If the personal or medication history was not conclusive, the participants' primary care physician was contacted for verification. All participants provided written informed consent before participating in the study.

\section{Reagents and Chemicals}

We used the following reagents: Ficoll-Paque PLUS (Ficoll; no. 17-1440-02; GE Healthcare, Uppsala, Sweden); 2-(4-iodophenyl)3-(4-nitrophenyl)-5-(2,4-disulfophenyl)-2H-tetrazolium (WST-1; no. 150849-52-8; Dojindo Laboratories, Kumamoto, Japan); interferon- $\gamma$ (IFN- $\gamma$; no. PHC4031; Invitrogen, Basel, Switzerland), tumor necrosis factor- $\alpha$ (TNF- $\alpha$; no. PHC3016; Invitrogen); Hank's balanced salt solution without phenol red (HBSS; no. 14025050; Invitrogen); fetal bovine serum (FBS; no. 10106169; Invitrogen); lipopolysaccharide (LPS; no. L6529; SigmaAldrich, Buchs, Switzerland); phosphate buffered saline (PBS; no. P5368; Sigma-Aldrich); phorbol 12-myristate 13-acetate (PMA; no. P8139; Sigma-Aldrich); RPMI-1640 medium with glutamax (RPMI-1640; no. W9925E; Fisher Scientific, Wohlen, Switzerland).

\section{Study Protocol}

Participants of the stress wound group and nonstress wound group reported to the laboratory by 10 a.m. and had abstained from extensive physical exercise, alcohol, and caffeinated beverages during the previous $24 \mathrm{hr}$. Participants were given a calorically standardized breakfast with comparable nutritional composition before an indwelling venous catheter was inserted not only for blood sampling but also to induce an open wound. The following resting period of $165 \mathrm{~min}$ was intended to allow in vivo stimulation of circulating monocytes by our applied open wound paradigm (Kuebler, Wirtz et al., 2013). Notably, we previously validated our open wound paradigm by comparing macrophage activity based on blood samples obtained by short-term cannula insertion with macrophage activity based on blood samples obtained by long-term catheter insertion (for details, see Kuebler, Wirtz et al., 2013). Moreover, our open wound paradigm is supported by previous findings suggesting cytokine-stimulating effects of intravenous catheter insertion (Haack et al., 2000). Next, participants of the stress wound group were exposed to the TSST (Kirschbaum et al., 1993), which comprises a short introduction followed by a 3-min preparation period, a 5-min mock job interview, and a 5-min mental arithmetic task (serial subtraction) in front of an unknown panel of two persons. At the end of the preparation phase and immediately before the beginning of the mock job interview, participants of the stress wound group completed the self-report Primary Appraisal Secondary Appraisal (PASA) scale (see below). In contrast, participants of the nonstress wound group were told in a nonstress introduction that they were required to stand in an empty room for $10 \mathrm{~min}$. This standing period in parallel to the orthostatic position during the TSST was intended to control for a possible confounding influence of orthostatic stress. Moreover, participants of the 


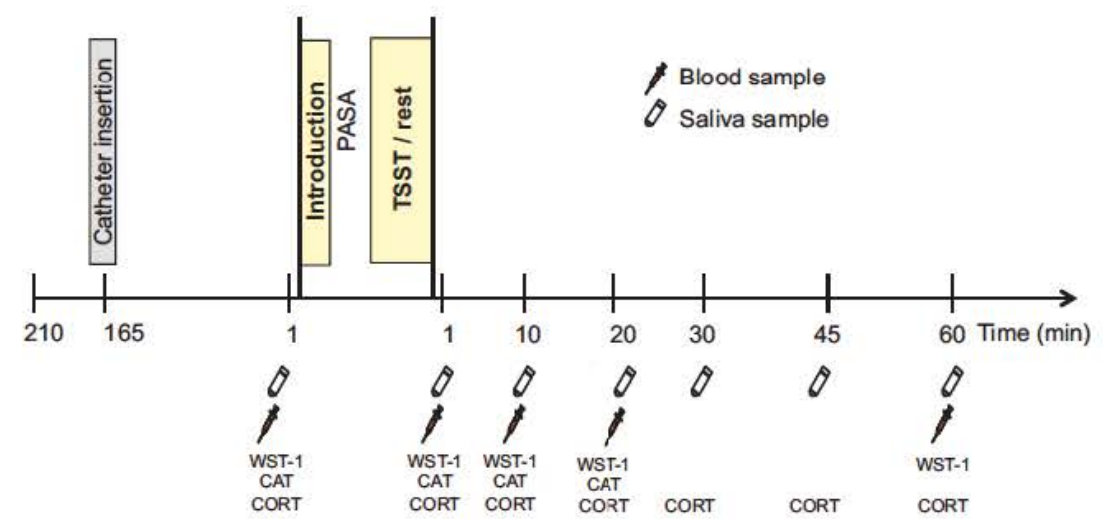

Figure 1. Experimental protocol. PASA = Primary Appraisal Secondary Appraisal scale; TSST $=$ Trier Social Stress Test; CAT = catecholamines (i.e., norepinephrine, epinephrine); CORT $=$ cortisol.

nonstress wound group completed the PASA scale at the same time as participants of the stress wound group. Before and after TSST/ rest, participants remained seated in a quiet room.

Blood samples for WST-1 reduction measurements were obtained immediately before (serving as baseline levels) and 1, 10, and $60 \mathrm{~min}$ after TSST/rest cessation. To determine catecholamine levels, blood samples were taken immediately before (baseline) and 1,10 , and $20 \mathrm{~min}$ after TSST/rest cessation. For determination of salivary-free cortisol levels, samples of saliva were collected immediately before (baseline) and 1, 10, 20,30, 45, and $60 \mathrm{~min}$ after TSST/rest cessation (see Figure 1). To determine mean arterial blood pressure (MAP), blood pressure (BP) was measured by sphygmomanometry (Omron 773; Omron Healthcare Europe B.V., Hoofdorp, Netherlands) immediately before and $40 \mathrm{~min}$ after insertion of the venous catheter. MAP was calculated by the formula $(2 / 3$ mean diastolic BP $)+(1 / 3$ mean systolic BP $)$. At the end of the experiment, participants were debriefed and participation was remunerated with 175 Swiss francs.

\section{Psychological Assessment}

Transactional stress questionnaire. Anticipatory cognitive stress appraisal processes were measured by self-report using the 16-item questionnaire for primary and secondary appraisal (PASA; Gaab, 2009; Gaab et al., 2005). The PASA was designed to fit with the respective description of the transactional stress model proposed by Lazarus and Folkman (1984). The PASA comprises the scales "primary appraisal" (PA) and "secondary appraisal" (SA), as well as the global PASA scale, termed "stress index." The PASA primary appraisal scale comprises the dimensions "threat" and "challenge" and refers to a person's judgment about the significance of an event as stressful, positive, controllable, challenging, or irrelevant. The PASA secondary appraisal scale combines the dimensions "selfconcept" and "control expectancy" and assesses available coping resources and options when faced with the stressor. Each of the PASA scales comprises eight items that are rated on a 6-point Likert scale (ranging from $1=$ strongly disagree to $6=$ strongly agree). The global PASA stress index scale combines the two PASA scales (i.e., primary and secondary appraisal) by the following formula: (primary appraisal: threat + challenge) - (secondary appraisal: self-concept + control expectancy). The stress index provides an integrated measure of transactional stress perception with a possible score range of -20 to 20 . Higher scores in the stress index mean higher stress appraisal. The questionnaire has good psychometric properties (Gaab et al., 2005) and Cronbach's alphas of our sample $(N=39)$ were .81 (primary appraisal) and .81 (secondary appraisal). PASA data were analyzed according to the analysis instructions proposed by the author (Gaab, 2009).

Chronic stress questionnaire. Chronic stress was measured using the 12-item Chronic Stress Screening Scale (CSSS; Schulz, Schlotz, \& Becker, 2004). The CSSS assesses the frequency of experiencing work overload (4 items), worries (4 items), lack of social recognition ( 2 items), excessive demands at work ( 1 item), and social overload ( $1 \mathrm{item})$. The items have a 5 -point rating format reflecting frequency (ranging from $0=$ never to $4=$ very often). Higher scores mean higher chronic stress. The questionnaire has good psychometric properties (Schulz et al., 2004), and Cronbach's alpha in our sample was .90 .

\section{Physiological Assessment}

WST-1 macrophage assay. The method used to study microbicidal potential of ex vivo isolated HMDM is based on that described by Kuebler and colleagues (Kuebler, Ehlert et al., 2013). In brief, $9 \mathrm{ml}$ of blood were collected in EDTA-coated tubes (Sarstedt, Numbrecht, Germany), immediately layered on top of $10 \mathrm{ml}$ Ficoll, and centrifuged for $20 \mathrm{~min}$ at $300 \times g$ and $20^{\circ} \mathrm{C}$. After centrifugation, peripheral blood mononuclear cells (PBMCs) were removed from the interface, washed twice in RPMI-1640 medium, counted with a hematologic analyzer (KX-21N; Sysmex Digitana AG), and resuspended to a concentration of $2.5 \times 10^{6} / \mathrm{ml}$ with RPMI-1640 medium supplemented with $10 \%$ FBS. Then, PBMC suspension aliquots of $1 \mathrm{ml}$ were transferred to 24-well cell culture plates (no. 4609; Semadeni, Ostermundigen, Switzerland). After incubation for $1 \mathrm{hr}$ at $37^{\circ} \mathrm{C}$ and $5 \% \mathrm{CO}_{2}$, the supernatant was discarded and the plate surface was rinsed five times with $1 \mathrm{ml}$ of warm $\left(25^{\circ} \mathrm{C}\right) 0.01 \mathrm{M}$ PBS to remove nonadherent PBMCs, while monocytes remained adherent to the bottom of the plate.

The adherent monocyte layer was diluted with $1 \mathrm{ml}$ RPMI-1640 medium supplemented with $10 \%$ FBS. Subsequently, we added $5 \mu \mathrm{l}$ IFN- $\gamma, 2 \mu \mathrm{l} \mathrm{TNF}-\alpha$, and $0.5 \mu \mathrm{l}$ LPS resulting in a final concentration of $50 \mathrm{ng} / \mathrm{ml} \mathrm{IFN}-\gamma, 20 \mathrm{ng} / \mathrm{ml} \mathrm{TNF}-\alpha$, and $300 \mathrm{ng} / \mathrm{ml}$ LPS to promote differentiation of monocytes into inflammatory macrophages (Gordon \& Taylor, 2005; Martinez, Sica, Mantovani, \& Locati, 2008). After incubation for $48 \mathrm{hr}$ at $37^{\circ} \mathrm{C}$ and $5 \% \mathrm{CO}_{2}$, 
the supernatant was discarded and the adherent macrophage layer was washed three times with $1 \mathrm{ml}$ of warm $\left(25^{\circ} \mathrm{C}\right) 0.01 \mathrm{M}$ PBS to remove traces of culture media and nonadherent cells.

Next, the resulting macrophage monolayer (obtained as described above) was overlaid with $1 \mathrm{ml}$ HBSS. Subsequently, $2 \mu 1$ WST-1, $0.5 \mu$ LPS, $5 \mu$ IFN- $\gamma, 2 \mu$ TNF- $\alpha$, and $0.5 \mu$ PMA were added, resulting in a final concentration of $100 \mu \mathrm{M}$ WST-1, $300 \mathrm{ng} / \mathrm{ml} \mathrm{LPS}, 50 \mathrm{ng} / \mathrm{ml}$ IFN- $\gamma, 20 \mathrm{ng} / \mathrm{ml}$ TNF- $\alpha$, and $50 \mathrm{nM}$ PMA. This was followed by an incubation period of $4 \mathrm{hr}$ at $37^{\circ} \mathrm{C}$ and $5 \% \mathrm{CO}_{2}$. Then, the supernatant was removed and used to determine WST-1 reduction by reading the optical density (OD) with a spectrophotometer (SmartSpec Plus, Bio-Rad Laboratories, Inc.) at $450 \mathrm{~nm}$ against water as blank. Higher ODs as obtained in absorbance reading are associated with higher amounts of reduced WST-1 and thus of superoxide anions generated by HMDM.

Stress hormone assays. For NE and epinephrine (EPI) assessment, venous blood was drawn in EDTA-coated monovettes (Sarstedt, Numbrecht, Germany), and immediately centrifuged for $10 \mathrm{~min}$ at $2,000 \times \mathrm{g}$ and $4^{\circ} \mathrm{C}$. Obtained plasma was stored at $-80^{\circ} \mathrm{C}$ until analysis. Plasma NE and EPI levels were determined by means of high-pressure liquid chromatography (HPLC) and electrochemical detection after liquid-liquid extraction (Ehrenreich et al., 1997; Smedes, Kraak, \& Poppe, 1982) in the Laboratory for Stress Monitoring (Göttingen, Germany). For both NE and EPI, the detection limit was $6 \mathrm{pg} / \mathrm{ml}$, and inter- and intraassay coefficients of variance $(\mathrm{CVs})$ were $<5 \%$.

For salivary-free cortisol assessment, saliva samples were collected in Salivettes (Sarstedt, Sevelen, Switzerland) and stored at $-20^{\circ} \mathrm{C}$ until analysis. Centrifugation of thawed saliva samples was at $2000 \times$ g, yielding low-viscosity saliva. Free cortisol concentrations were determined using a commercial chemiluminescence immunoassay with high sensitivity of $0.16 \mathrm{ng} / \mathrm{ml}$ (IBL, Hamburg, Germany). Inter- and intraassay CVs were $<11.5 \%$ and $7.7 \%$, respectively.

\section{Statistical Analyses}

Data were analyzed using IBM SPSS Statistics version 20.0 for Mac OS X and presented as mean $\pm S E M$. All tests were two-tailed with the significance level set at $p \leq .05$ and the level of borderline significance set at $p \leq .10$. All data were tested for normal distribution and homogeneity of variance using Kolmogorov-Smirnov and Levene's tests prior to statistical analyses. No outliers were excluded. Skewed EPI values were logarithmically transformed, and normal distribution was verified. We calculated body mass index (BMI) as the weight in kilograms divided by height in meters squared. Stress hormone changes were determined by calculating the difference between the respective peak stress response (based on the maximum mean response of the respective parameter observed in the stress group) minus baseline level. NE and EPI changes $(\triangle \mathrm{NE}, \Delta \mathrm{EPI})$ were calculated as the difference in plasma levels between $1 \mathrm{~min}$ post-TSST/rest and baseline. Cortisol stress changes $(\triangle \mathrm{CORT})$ refer to changes from baseline to $20 \mathrm{~min}$ postTSST/rest. Due to high levels of interindividual variations, we followed previous research (Ellard, Castle, \& Mian, 2001) and calculated macrophage microbicidal potential as percentage changes in repeatedly measured WST-1 reduction scores in relation to the respective baseline. For repeatedly measured WST-1 reduction, area under the total response curve with respect to ground (AUC) was calculated with the trapezoid formula (Pruessner, Kirschbaum, Meinlschmid, \& Hellhammer, 2003). Based on our previous observations on associations between PASA scales and stress reactivity of immune measures (Wirtz, von Kanel et al., 2007), we expected a medium effect size to detect an interaction effect between stress appraisal and stress reactivity of our immune measure. $\mathrm{G}^{*}$ power 3.1 analysis suggests that a total sample size of $N=32$ would be sufficient to detect an interaction effect between stress appraisal and stress reactivity of HMDM in two groups with an expected medium effect size of $f=.25$ in general models with repeated measures with a power of $.90, \alpha=.05$, given the observed correlations of the repeated measures of $\geq .47$.

To calculate differences between the characteristics of the stress wound group and the nonstress wound group, we used univariate analyses of variance (ANOVAs). In order to test whether the TSST induced a significant HMDM and neuroendocrine stress response, we calculated repeated measures analyses of covariance (ANCOVAs) with group (stress wound group vs. nonstress wound group) as the independent variable and the four and seven time points in which reduced WST-1, catecholamine (NE and EPI), or cortisol were measured as repeated dependent variable. Notably, although results of these calculations in the initial total study group of 41 subjects have been previously reported (Kuebler, Wirtz et al., 2013), we here recalculate them in the 39 subjects with stress appraisal data.

To test our main hypothesis, that is, whether the previously observed inhibitory effect of stress on wound-induced macrophage activation relates to cognitive stress appraisal processes in terms of primary and secondary stress appraisal, (Step 1) we calculated general linear models with repeated measures of WST-1 reduction as dependent variables and stress appraisal as linear independent variable. Based on our hypothesis that stress appraisal would relate to WST-1 reduction in the stress wound group but not in the nonstress wound group, significant associations were then further analyzed by (Step 2) repeating the initial calculation in the stress wound group and the nonstress wound group separately. To further explore whether significant associations between stress appraisal and WST-1 reduction would differ between groups, (Step 3) we calculated multiple regression analyses (Baron \& Kenny, 1986; Frazier, Tix, \& Barron, 2004). As the dependent variable, we entered AUC of WST-1 reduction. As independent variables, we entered stress appraisal, stress-induction group (stress wound group vs. nonstress wound group), and the interaction between stress appraisal and group. A significant interaction term would indicate that associations between stress appraisal and WST-1 reduction differ between groups. Prior to regression analyses, all predictor and modulator variables were $Z$ transformed. To account for the lower power of AUC given our sample size, we repeated the analyses of Step 3 in a complementary repeated measures ANCOVA approach (repeated WST-1 reduction as dependent variable; the interaction term and all other predictors from the regression approach as independent variables).

To graphically illustrate and interpret our results, we followed Frazier et al. (2004) and plotted WST-1 reduction AUC scores for nonstressed and stressed participants at the mean, and at low $(-1$ $S D$ from the mean) and high (1 SD from the mean) levels of cognitive stress appraisal. Moreover, to take into account that these mean, low, and high levels of cognitive stress appraisal differ between our subject groups, we additionally split the stress wound group and nonstress wound group into four representative subject groups based on their observed level in significant PASA scales (stress wound group/lower PASA scores [PA: 1.5 to $3.4, n=12$; stress: $>-5$ to $-0.5, n=11]$; stress wound group/higher PASA scores [PA: $>3.4, n=10$; stress: $>-0.5, n=11]$; nonstress wound 
Table 1. Biological and Psychological Characteristics of the Study Participants

\begin{tabular}{|c|c|c|c|}
\hline & Stress wound group & Nonstress wound group & \\
\hline & $(n=22)$ & $(n=17)$ & $p$ ANOVA \\
\hline Age (year) & $35.82 \pm 2.0(20-50)$ & $34.7 \pm 1.9(22-49)$ & .68 \\
\hline BMI $\left(\mathrm{kg} / \mathrm{m}^{2}\right)$ & $24.7 \pm 0.9(18.7-36.0)$ & $24.6 \pm 0.5(22.5-30.1)$ & .96 \\
\hline MAP (mm Hg) & $89.4 \pm 1.9(75.8-105.5)$ & $87.8 \pm 1.3(80.8-102.5)$ & .52 \\
\hline NE baseline (pg/ml) & $452.6 \pm 41.1(115.3-841.7)$ & $419.2 \pm 48.5(166.2-765.6)$ & .60 \\
\hline EPI baseline (pg/ml) & $21.3 \pm 3.3(6.1-79.1)$ & $20.9 \pm 3.5(7.1-47.3)$ & .85 \\
\hline Cortisol baseline (nmol/l) & $5.3 \pm 0.6(2.9-13.7)$ & $3.7 \pm 0.4(1.6-8.2)$ & .039 \\
\hline$\Delta \mathrm{NE}(\mathrm{pg} / \mathrm{ml})$ & $226.7 \pm 25.2(25.6-511.0)$ & $64.1 \pm 23.0(-109.5-212.9)$ & $<.001$ \\
\hline$\Delta \mathrm{EPI}(\mathrm{pg} / \mathrm{ml})$ & $43.3 \pm 8.4(5.3-157.5)$ & $4.5 \pm 2.3(-13.9-20.2)$ & .001 \\
\hline$\Delta \mathrm{CORT}(\mathrm{nmol} / \mathrm{l})$ & $16.6 \pm 2.1(-1.0-40.5)$ & $0.7 \pm 0.8(-1.6-12.3)$ & $<.001$ \\
\hline PASA primary appraisal & $3.4 \pm 0.13(2.3-5.13)$ & $1.7 \pm 0.14(1.0-3.0)$ & $<.001$ \\
\hline PASA secondary appraisal & $3.7 \pm 0.19(1.6-5.1)$ & $4.3 \pm 0.25(2.3-6.0)$ & .055 \\
\hline PASA stress index & $-0.5 \pm 0.48(-4.5-4.8)$ & $-5.2 \pm 0.55(-10.0-1.8)$ & $<.001$ \\
\hline Chronic stress (CSSS) & $9.9 \pm 1.48(1-25)$ & $11.4 \pm 1.63(1-29)$ & .51 \\
\hline
\end{tabular}

Note. Values are given as means \pm SEM (range). $\mathrm{BMI}=$ body mass index; MAP $=$ mean arterial blood pressure; $\mathrm{NE}=$ norepinephrine; EPI = epinephrine; $\mathrm{CORT}=\operatorname{cortisol} ; \Delta=$ stress hormone change; PASA = Primary Appraisal Secondary Appraisal scale; CSSS = Chronic Stress Screening Scale.

group/lower PASA scores [PA: $<1.5, n=8$; stress: $\leq-5, n=7$ ]; nonstress wound group/higher PASA scores [PA: 1.5 to $3.4, n=9$; stress: $>-5$ to $-0.5, n=10])$. Notably, this grouping procedure was used for graphic illustration only, but not for modeling and testing.

Finally, to explore potential underlying mechanisms of significant associations between cognitive stress appraisal measure(s) and WST-1 reduction, we first tested whether stress changes of norepinephrine $(\Delta \mathrm{NE})$, epinephrine $(\Delta \mathrm{EPI})$, and cortisol $(\Delta \mathrm{CORT}) \mathrm{sig}$ nificantly relate to repeated WST-1 reduction using general linear models. Significant associations were further tested for potential mediation effects. According to Baron and Kenny (1986), statistical mediation holds if (Step 1) the independent variable (PASA scale) is associated with the supposed mediator (i.e., $\triangle \mathrm{NE}, \triangle \mathrm{EPI}$, $\triangle \mathrm{CORT}$ ), if (Step 2) the independent variable (PASA scale) is associated with the dependent variable (repeated WST-1 reduction), and if (Step 3) the mediator (i.e., $\Delta \mathrm{NE}, \Delta \mathrm{EPI}, \Delta \mathrm{CORT}$ ) is significantly associated with the dependent variable (repeated WST-1 reduction) while controlling for the independent variable (PASA scale) and the association between the independent variable (PASA scale), and the dependent variable (WST-1 reduction) is lower than in Step 2 (Baron \& Kenny, 1986). We favored ANCOVAs over multiple linear regression analyses to test for statistical mediation because Steps 2 and 3 include repeatedly measured WST-1 reduction as the dependent variable. The use of multiple linear regression analysis would require artificial integration of the repeatedly measured dependent variable into a single measure index resulting in a loss of information.

Post hoc testing of significant mediation effects comprised statistical evaluation of the indirect effect. To do this, we followed Preacher and Hayes (2008) and performed nonparametric bootstrapping analyses with $n=5,000$ resamples and $95 \%$ biascorrected and accelerated (BCa) confidence intervals $(\mathrm{CI})$. In these analyses, mediation is significant if zero is not included in the 95\% $\mathrm{BCa}$ CI. Because bootstrapping analyses require single measure indices, AUC of WST-1 reduction was entered as the dependent variable.

Since previous literature suggests a potential influence of age, BMI, and MAP on microbicidal potential of HMDM (Dorshkind, Montecino-Rodriguez, \& Signer, 2009; Grossman, 2008; Wirtz, Ehlert, Emini, \& Suter, 2008), we controlled for age, BMI, and MAP in all WST-1 reduction analyses. In secondary analyses, we additionally controlled for an a priori defined set of potential confounders (i.e., baseline cortisol levels and chronic stress; Dhabhar, 2014).

Effect size parameters $(f)$ were calculated from partial $\eta^{2}$ values and are reported where appropriate (effect size conventions, $f: .10=$ small, $.25=$ medium, $.40=$ large $)$.

\section{Results}

\section{Participant Characteristics}

Table 1 provides the sociodemographic, biological, and psychological characteristics of the 39 participants with complete cognitive stress appraisal assessment. The two groups did not significantly differ in age, BMI, MAP, NE, or EPI baseline levels ( $p$ s > .51). Cortisol baseline levels were higher in the stress wound group than in the nonstress wound group. In addition, stressed participants had higher stress hormone changes $(\Delta \mathrm{EPI}, \Delta \mathrm{NE}$, $\Delta$ CORT) than nonstressed participants. Furthermore, compared with nonstressed participants, participants of the stress wound group showed significantly higher scores in the tertiary PASA stress index scale, as well as in the PASA primary appraisal scale. No group differences were observed in the PASA secondary appraisal scale or the CSSS scale. Due to technical problems, NE and EPI data from two participants of the nonstress wound group were missing.

\section{Manipulation Checks}

Repeated measures of microbicidal potential of macrophages in terms of WST-1 reduction was suppressed in the stress wound group as compared to the nonstress wound group (main effect of group: $F(1,34)=6.52, p=.015, f=.43$, see Figure 2$)$. While the nonstress wound group displayed a significant increase in WST-1 reduction over time (main effect of time: $F(3,39)=4.79, p=.006$, $f=.61)$, the stress wound group did not $(p=.75)$. In addition, stressed participants showed significant increases in all three stress hormones in reaction to TSST exposure compared with nonstressed participants (NE: Group $\times$ Time interaction: $F(3.0,96)=8.12, p<.001, f=.51$; EPI: Group $\times$ Time interaction: 


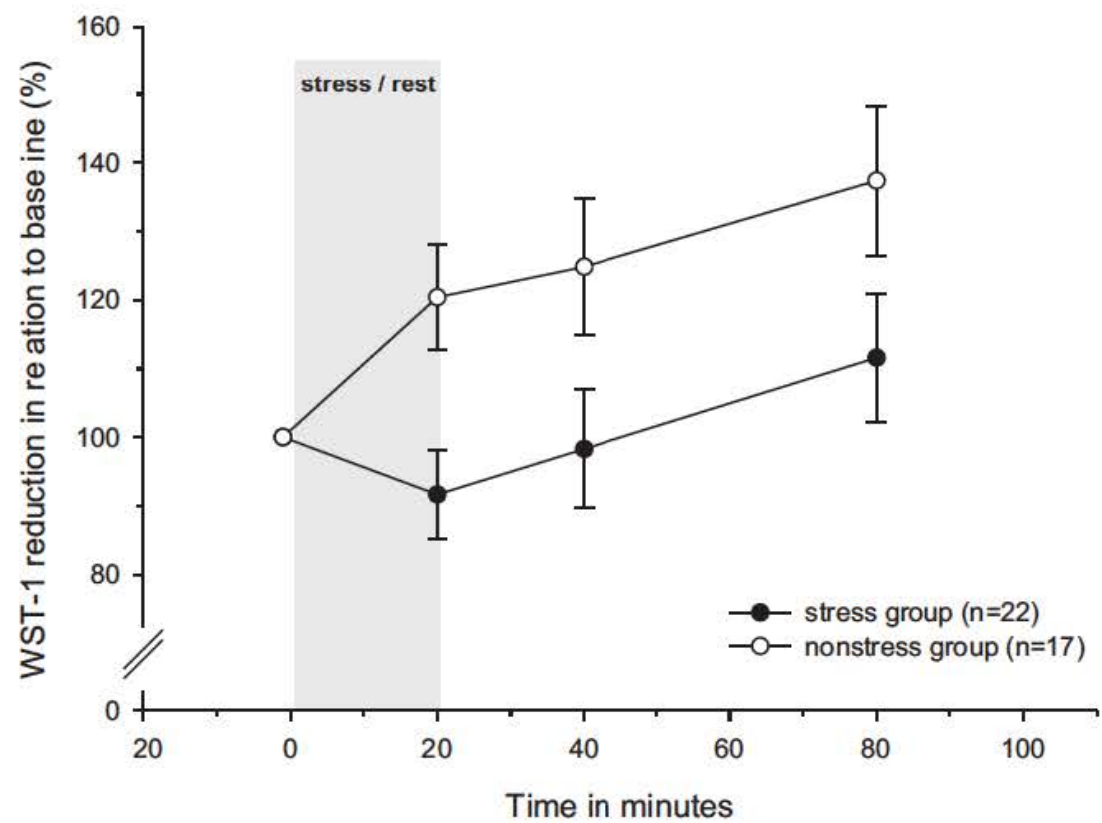

Figure 2. Course of WST-1 reduction over time in the stress wound group and the nonstress wound group. Values are given as means \pm SEM.

$F(3.0,96)=21.57, p<.001, f=.82$; cortisol: Group $\times$ Time interaction: $F(3.0,102)=27.52, p<.001, f=.90)$. Age, BMI, and MAP were controlled.

\section{Stress Appraisal and Microbicidal Potential of Macrophages}

Step 1. Testing for associations between repeatedly measured macrophage microbicidal potential in terms of WST-1 reduction and cognitive stress appraisal revealed that WST-1 reduction over time was significantly associated with the global PASA stress index scale (main effect: $F(1,34)=5.26, p=.028, f=.39$ ), the PASA primary appraisal scale (main effect: $F(1,34)=7.98, p=.008$, $f=.48)$, but not the PASA secondary appraisal scale $(p=.56)$.

Step 2. In the stress wound group but not in the nonstress wound group $(p=.25)$, repeatedly measured WST-1 reduction was significantly associated with the PASA primary appraisal scale (main effect: $F(1,17)=11.13, p=.004, f=.81)$. In terms of the PASA stress index scale, the association with repeatedly measured WST-1 reduction in the stress wound group became of borderline significance (main effect: $F(1,17)=3.77, p=.069, f=.47$ ). Similar to primary appraisal, no association was found for the stress index in the nonstress wound group $(p=.18)$.

Step 3. The interaction between primary appraisal and group (stress wound group vs. nonstress wound group) significantly predicted AUC of WST-1 reduction (Primary Appraisal $\times$ Group: $\beta=-.363, p=.007, \Delta R^{2}=.12$, see Figure 3); independent of MAP, BMI, age, primary appraisal, and group with the total model explaining $54 \%$ of the variance in WST-1 reduction AUC $\left(R^{2}=0.54 ; F(6 / 38)=6.22, p<.001\right)$. Independent of MAP, BMI, age, stress index, and group, the interaction between stress index and group was of borderline significance in predicting WST-1 reduction AUC (Stress Index $\times$ Group: $\beta=-.261, p=.070$, $\Delta R^{2}=.059$, see Figure 4 ). The whole model explained $46 \%$ of the variance in WST-1 reduction AUC $\left(R^{2}=0.46 ; F(6 / 38)=4.54\right.$, $p=.002$ ). Complementary ANCOVA results confirmed that both the PASA primary appraisal scale and the global PASA stress index scale independently related to the observed stress-induced attenuation of WST-1 reduction over time (main effect of Group $\times$ Primary Appraisal interaction: $F(1 / 32)=8.10, p=.008$, $f=.50$; main effect of Group $\times$ Stress Index interaction: $F(1 /$ $32)=3.96, p=.055, f=.35$ ).

Additional controlling for baseline cortisol levels and chronic stress levels (i.e., CSSS scores) did not significantly change results.

\section{Stress Hormone Responses, Stress Appraisal, and} Microbicidal Potential of Macrophages

Only the NE stress response $(\Delta \mathrm{NE} ; F(1 / 31)=5.80, p=.022$, $f=.43$ ) significantly related to repeated WST-1 reduction, while $\triangle$ EPI and $\triangle$ CORT did not ( $p s>.42$ ).

Statistical mediation testing revealed that $\triangle \mathrm{NE}$ significantly mediated the observed relationship between the PASA scale primary appraisal and WST-1 reduction: (a) $\triangle \mathrm{NE}$ was independently predicted by primary appraisal $\left(\beta=.66, p<.001, \Delta R^{2}=.40\right)$ and (b) repeated WST-1 reduction was independently predicted by $\Delta \mathrm{NE}, F(1 / 32)=12.2, p<.001, f=.62$. In addition, controlling for $\triangle \mathrm{NE}$ revealed that primary appraisal was no longer associated with repeated WST-1 reduction $(p=.24)$, while $\Delta \mathrm{NE}$ was, although of borderline significance, $F(1 / 31)=3.67, p=.065, f=.34$. Similar results were found for the PASA scale stress index: $\triangle \mathrm{NE}$ was independently predicted by stress index $(\beta=.55, p=.001$, $\Delta R^{2}=.28$ ). In addition, controlling for $\Delta \mathrm{NE}$ revealed that stress index was no longer associated with repeated WST-1 reduction $(p=.50)$, while $\Delta \mathrm{NE}$ was $[F(1 / 31)=6.58, p=.015, f=.46]$.

The post hoc nonparametric bootstrapping analyses confirmed $\triangle \mathrm{NE}$ as mediator between the two PASA scales and WST-1 reduction (primary appraisal: $b=-53.73, \mathrm{BCa} 95 \% \mathrm{CI}:-134.90$ to -0.18 ; stress index: $b=-17.79$, $\mathrm{BCa} 95 \% \mathrm{CI}:-39.12$ to -4.29 ). 
A

Group x Primary Appraisal Interaction

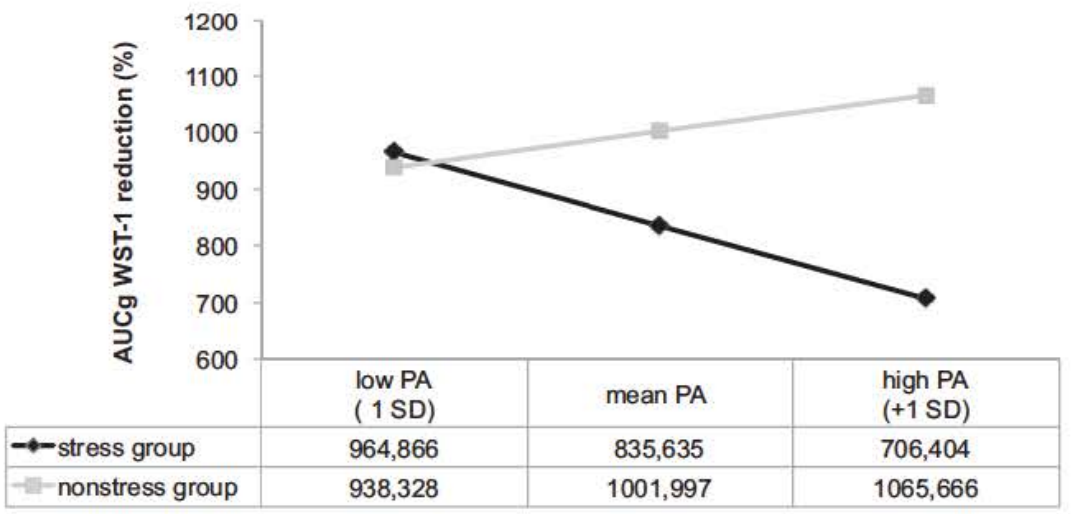

B

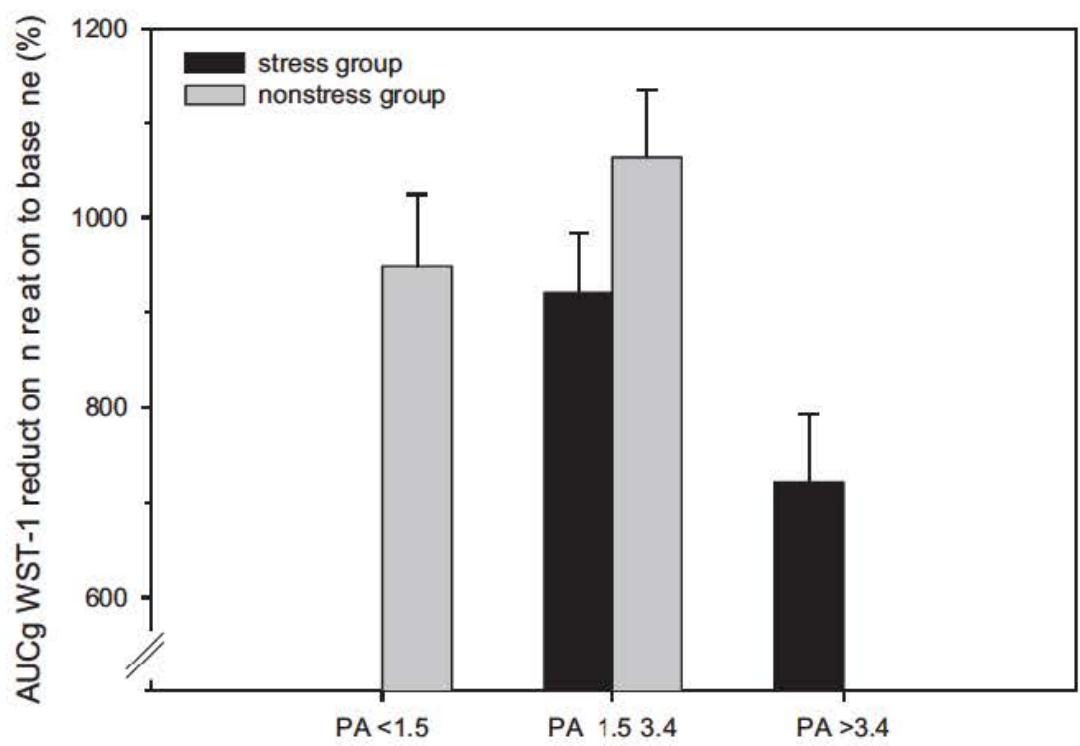

Figure 3. Effect of the PASA primary appraisal (PA) scale on wound-induced macrophage microbicidal potential in the stress wound group and the nonstress wound group. A: Plot of significant Group $\times$ Primary appraisal interaction. B: Area under the curve with regard to ground. Values are given as means \pm SEM.

\section{Discussion}

Our study investigates for the first time whether anticipatory cognitive stress appraisal modulates wound-induced activation of macrophage microbicidal potential in acutely stressed compared to nonstressed men. Despite the huge number of studies demonstrating the wound healing delaying effect of stress (Gouin \& Kiecolt-Glaser, 2011; Walburn et al., 2009), the effect of stress on noncirculating human macrophages has not yet been investigated. Our study is thereby the first that assesses associations with noncirculating but tissue-based human macrophages. As hypothesized, we found that in acutely stressed men, but not in nonstressed men, higher scores in anticipatory cognitive stress appraisal as measured by the PASA primary appraisal scale (combining threat and challenge) and by the global PASA stress index scale (combining primary appraisal and secondary appraisal) were associated with lower WST-1 reduction scores of PMA-activated HMDM following catheter-induced wound application. These results were of large effect size and independent of common correlates of immune cell activity. Given the anticipatory nature of stress appraisal assessment, these associative findings suggest that cognitive stress appraisal or primary appraisal, determines inhibitory effects on wound-induced WST-1 reduction. In contrast, the PASA secondary appraisal scale (combined self-concept and control expectancy) did not relate to repeatedly measured WST-1 reduction scores.

Our data suggest that it was not the participants' perception as to whether their resources would be sufficient to cope with the stressor, but rather their consideration of the stressor as threatening and/or challenging that was associated with the stress-induced suppression of wound-induced activation of macrophage microbicidal potential. In other words, it seems to be more the interpretation of a given event as threatening and/or challenging than the perceived competence and control over the event that determines the extent to which wound-induced microbicidal potential of macrophages is suppressed by stress. Given the observed statistical associations 
A

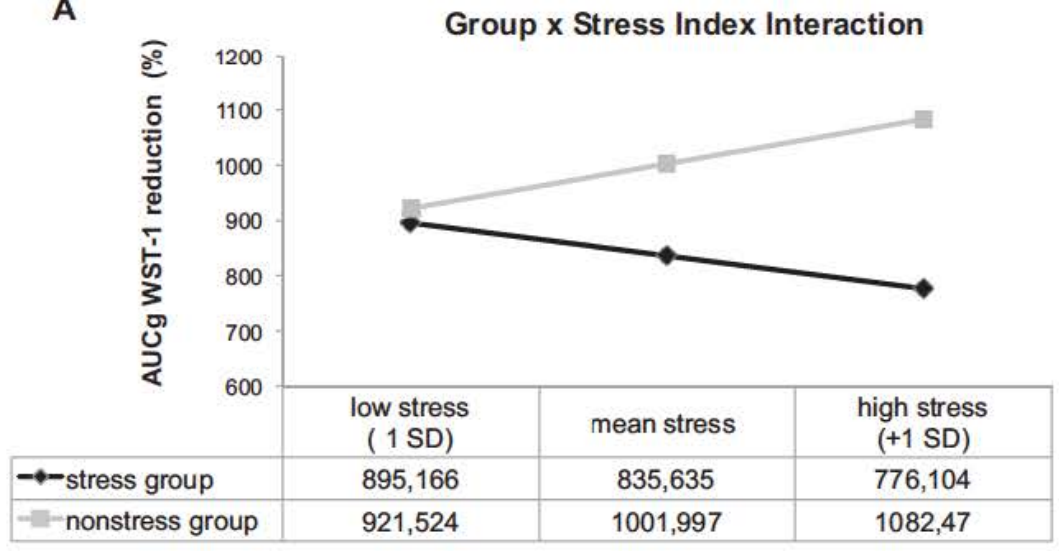

B

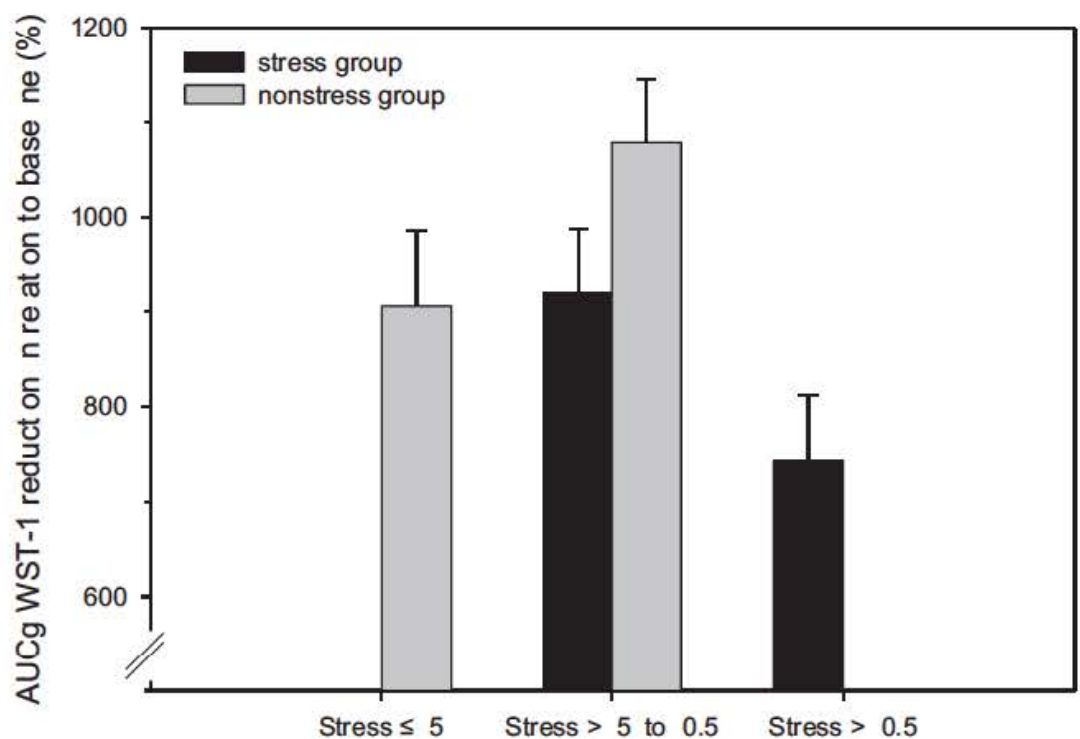

Figure 4. Effect of the global PASA stress index (stress) scale on wound-induced macrophage microbicidal potential in the stress wound group and the nonstress wound group. A: Plot of significant Group $\times$ Stress index interaction. B: Area under the curve with regard to ground. Values are given as means $\pm S E M$.

and the sequence of data assessment, our data further suggest the NE stress response to underlie the inhibitory effect of anticipatory cognitive stress appraisal on wound-induced macrophage activation. Notably, to the best of our knowledge, our data provide first evidence of a modulatory role of cognitive stress appraisal in the NE stress response, although cognitive stress appraisal has been established as an important determinant of the cortisol stress response (Gaab et al., 2005; Schlotz et al., 2011).

What are the potential clinical implications of our study? In light of the importance of microbicidal active M1-like macrophages for nondelayed skin wound healing (Lucas et al., 2010; Mori et al., 2004; Nagaoka et al., 2000; Rojas et al., 2002; Subramaniam et al., 1997), our findings may suggest that, in particular, individuals who anticipate a given stressful situation as more threatening and more challenging are more susceptible to stress-induced delays in skin wound healing. Thus, one might speculate that cognitive training directed at supporting individuals to perceive a stressful event as less threatening or challenging could provide psychophysiological benefit with respect to woundinduced activation of macrophage microbicidal potential and thus wound healing. Although the attenuating effect of cognitivebehavioral stress inoculation programs on both cognitive stress appraisal and the cortisol response to acute psychosocial stress has been repeatedly demonstrated (Gaab et al., 2003; Gaab, Sonderegger, Scherrer, \& Ehlert, 2006; Hammerfald et al., 2006), intervention studies have not yet been performed in the field of tissue-based immune cell functions. Future intervention studies are needed to show whether the suppression of wound-induced microbicidal potential of macrophages by acute psychosocial stress can be modified by improving coping skills and whether these effects could benefit patients with impaired wound healing.

Our study has several strengths: In addition to the use of our standardized and simple wound application paradigm, which seems 
to initiate wound healing processes in a rather natural manner (Kuebler, Wirtz et al., 2013), a further strength was the use of the innovative WST-1 macrophage assay (Kuebler, Ehlert et al., 2013). The WST-1 macrophage assay allows the study of M1 macrophages in vitro (Gordon \& Taylor, 2005; Martinez et al., 2008), a macrophage phenotype that in vivo is likely to be the dominant immune cell subtype in the wound area in early phases of wound healing (Engelhardt et al., 1998; Mahdavian Delavary et al., 2011; Martinez et al., 2008; Stroncek \& Reichert, 2008). We also used a highly standardized and potent stress test that reliably induces neuroendocrine stress responses (Campbell \& Ehlert, 2012; Dickerson \& Kemeny, 2004; Kirschbaum et al., 1993). Furthermore, we controlled for age, BMI, MAP, baseline cortisol levels, and chronic stress levels as a priori selected control variables to rule out potential confounding influences on microbicidal potential of HMDM. In addition, cognitive appraisal was assessed prior to changes in macrophage microbicidal potential following stress, which enhances the probability to detect causal relationships. The study also has its limitations. First, our sample size was relatively small and therefore our data should be interpreted with caution until confirmed in larger studies. Second, our findings are restricted to the stress induction by means of TSST and to healthy men. The generalizability of our findings to other types of stressors (e.g., chronic stress), or to patients suffering from a disease, or women, still needs to be confirmed by future research. Third, in our experiment the stress introduction that induced higher anticipatory stress appraisal was always followed by the announced stressor. Therefore, we are unable to determine whether our macrophage findings result from stress appraisal alone, from the stressor alone, or from their interaction. However, given that stress perception is conceptualized as an interaction between person and situation (Lazarus \& Folkman, 1984) and given that the situation (the TSST) was standardized for all participants of the stress group, we speculate that it is unlikely that the stress situation alone predicts macrophage activity, but rather that stress appraisal is likely to modulate stress reactivity. Whether stress appraisal is capable of modulating macrophage activity without stress induction needs to be investigated in future studies.

In sum, our data suggest that anticipatory cognitive stress appraisal relates to tissue-based immunity as we found it to modulate wound-induced activation of macrophage microbicidal potential in acutely stressed men, but not in nonstressed men. In particular, we observed stronger stress-induced suppression of wound-induced activation of macrophage microbicidal potential in men who perceived more threat and challenge in anticipation of the stressor. The NE stress response seems to underlie this finding. Future studies are needed to replicate our findings and to test potential clinical implications.

\section{References}

Baron, R. M., \& Kenny, D. A. (1986). The moderator-mediator variable distinction in social psychological research: Conceptual, strategic, and statistical considerations. Journal of Personality and Social Psychology, 51, 1173-1182.

Campbell, J., \& Ehlert, U. (2012). Acute psychosocial stress: Does the emotional stress response correspond with physiological responses? Psychoneuroendocrinology, 37, 1111-134. doi: 10.1016/j.psyneuen .2011 .12 .010

Daley, J. M., Brancato, S. K., Thomay, A. A., Reichner, J. S., \& Albina, J. E. (2010). The phenotype of murine wound macrophages. Journal of Leukocyte Biology, 87, 59-67.

Dhabhar, F. S. (2014). Effects of stress on immune function: The good, the bad, and the beautiful. Immunologic Research, 58, 193-210. doi: 10.1007/s12026-014-8517-0

Dickerson, S. S., \& Kemeny, M. E. (2004). Acute stressors and cortisol responses: A theoretical integration and synthesis of laboratory research. Psychological Bulletin, 130, 355-391. doi: 10.1037/00332909.130.3.355

Dorshkind, K., Montecino-Rodriguez, E., \& Signer, R. A. (2009). The ageing immune system: Is it ever too old to become young again? Nature Reviews Immunology, 9, 57-62. doi: 10.1038/nri2471

Ehrenreich, H., Schuck, J., Stender, N., Pilz, J., Gefeller, O., Schilling, L., ... Kaw, S. (1997). Endocrine and hemodynamic effects of stress versus systemic CRF in alcoholics during early and medium term abstinence. Alcoholism: Clinical and Experimental Research, 21, 12851293. doi: 10.1111/j.1530-0277.1997.tb04450.x

Ellard, D. R., Castle, P. C., \& Mian, R. (2001). The effect of a short-term mental stressor on neutrophil activation. International Journal of Psychophysiology, 41, 93-100.

Engelhardt, E., Toksoy, A., Goebeler, M., Debus, S., Brocker, E. B., \& Gillitzer, R. (1998). Chemokines IL-8, GROalpha, MCP-1, IP-10, and Mig are sequentially and differentially expressed during phase-specific infiltration of leukocyte subsets in human wound healing. American Journal of Pathology, 153, 1849-1860. doi: 10.1016/S00029440(10)65699-4

Frazier, P. A., Tix, A. P., \& Barron, K. E. (2004). Testing moderator and mediator effects in counselling psychology. Journal of Counselling Psychology, 51, 115-134.

Gaab, J. (2009). PASA-Primary appraisal secondary appraisal. Ein Fragebogen zur Erfassung von situationsbezogenen kognitiven Bewertungen [PASA — Primary appraisal secondary appraisal. A ques- tionnaire to assess anticipatory cognitive appraisal processes]. Verhaltenstherapie, 19, 114-115. doi: 10.1159/000223610

Gaab, J., Blattler, N., Menzi, T., Pabst, B., Stoyer, S., \& Ehlert, U. (2003). Randomized controlled evaluation of the effects of cognitive-behavioral stress management on cortisol responses to acute stress in healthy subjects. Psychoneuroendocrinology, 28, 767-779.

Gaab, J., Rohleder, N., Nater, U. M., \& Ehlert, U. (2005). Psychological determinants of the cortisol stress response: The role of anticipatory cognitive appraisal. Psychoneuroendocrinology, 30, 599-610. doi: 10.1016/j.psyneuen.2005.02.001

Gaab, J, Sonderegger, L., Scherrer, S., \& Ehlert, U. (2006). Psychoneuroendocrine effects of cognitive-behavioral stress management in a naturalistic setting-A randomized controlled trial. Psychoneuroendocrinology, 31, 428-438. doi: 10.1016/j.psyneuen .2005 .10 .005

Gordon, S., \& Taylor, P. R. (2005). Monocyte and macrophage heterogeneity. Nature Reviews Immunology, 5, 953-964. doi: 10.1038/nri1733

Gouin, J. P., \& Kiecolt-Glaser, J. K. (2011). The impact of psychological stress on wound healing: Methods and mechanisms. Immunology and Allergy Clinics of North America, 31, 81-93. doi: 10.1016/j.iac .2010 .09 .010

Grossman, E. (2008). Does increased oxidative stress cause hypertension? [Supplement 2]. Diabetes Care, 31, S185-S189. doi: 10.2337/dc08s246

Haack, M., Reichenberg, A., Kraus, T., Schuld, A., Yirmiya, R., \& Pollmacher, T. (2000). Effects of an intravenous catheter on the local production of cytokines and soluble cytokine receptors in healthy men. Cytokine, 12, 694-698. doi: 10.1006/cyto.1999.0665

Hammerfald, K., Eberle, C., Grau, M., Kinsperger, A., Zimmermann, A., Ehlert, U., \& Gaab, J. (2006). Persistent effects of cognitive-behavioral stress management on cortisol responses to acute stress in healthy subjects-A randomized controlled trial. Psychoneuroendocrinology, 31, 333-339. doi: 10.1016/j.psyneuen.2005.08.007

Het, S., Rohleder, N., Schoofs, D., Kirschbaum, C., \& Wolf, O. T. (2009). Neuroendocrine and psychometric evaluation of a placebo version of the "Trier Social Stress Test." Psychoneuroendocrinology, 34, 10751086. doi: 10.1016/j.psyneuen.2009.02.008

Kirschbaum, C., Kudielka, B. M., Gaab, J., Schommer, N. C., \& Hellhammer, D. H. (1999). Impact of gender, menstrual cycle phase, and oral contraceptives on the activity of the hypothalamus-pituitaryadrenal axis. Psychosomatic Medicine, 61, 154-162. 
Kirschbaum, C., Pirke, K. M., \& Hellhammer, D. H. (1993). The "Trier Social Stress Test"-A tool for investigating psychobiological stress responses in a laboratory setting. Neuropsychobiology, 28, 76-81. doi: $10.1159 / 000119004$

Kuebler, U., Ehlert, U., Zuccarella, C., Sakai, M., Stemmer, A., \& Wirtz, P. H. (2013). An in vitro method to investigate the microbicidal potential of human macrophages for use in psychosomatic research. Psychosomatic Medicine, 75, 841-848. doi: 10.1097/PSY.0000000000000008

Kuebler, U., Wirtz, P. H., Sakai, M., Stemmer, A., \& Ehlert, U. (2013). Acute stress reduces wound-induced activation of microbicidal potential of ex vivo isolated human monocyte-derived macrophages. PLoS One, 8, e55875. doi: 10.1371/journal.pone.0055875

Lazarus, R. S., \& Folkman, S. (1984). Stress, appraisal, and coping. New York, NY: Springer Publishing Company.

Lucas, T., Waisman, A., Ranjan, R., Roes, J., Krieg, T., Muller, W., .. . Eming, S. A. (2010). Differential roles of macrophages in diverse phases of skin repair. Journal of Immunology, 184, 3964-3977. doi: 10.4049/jimmunol.0903356

Mahdavian Delavary, B., van der Veer, W. M., van Egmond, M., Niessen, F. B., \& Beelen, R. H. (2011). Macrophages in skin injury and repair Immunobiology, 216, 753-762. doi: 10.1016/j.imbio.2011.01.001

Martinez, F. O., Sica, A., Mantovani, A., \& Locati, M. (2008). Macrophage activation and polarization. Frontiers in Bioscience, 13, 453-461.

Mori, R., Kondo, T., Nishie, T., Ohshima, T., \& Asano, M. (2004). Impairment of skin wound healing in beta-1,4-galactosyltransferase-deficient mice with reduced leukocyte recruitment. American Journal of Pathology, 164, 1303-1314. doi: 10.1016/S0002-9440(10)63217-8

Nagaoka, T., Kaburagi, Y., Hamaguchi, Y., Hasegawa, M., Takehara, K., Steeber, D. A., ... Sato, S. (2000). Delayed wound healing in the absence of intercellular adhesion molecule-1 or L-selectin expression. American Journal of Pathology, 157, 237-247. doi: 10.1016/S00029440(10)64534-8

Preacher, K. J., \& Hayes, A. F. (2008). Asymptotic and resampling strategies for assessing and comparing indirect effects in multiple mediator models. Behavior Research Methods, 40, 879-891.

Pruessner, J. C., Kirschbaum, C., Meinlschmid, G., \& Hellhammer, D. H. (2003). Two formulas for computation of the area under the curve represent measures of total hormone concentration versus timedependent change. Psychoneuroendocrinology, 28, 916-931.

Rojas, I. G., Padgett, D. A., Sheridan, J. F., \& Marucha, P. T. (2002) Stress-induced susceptibility to bacterial infection during cutaneous wound healing. Brain, Behavior, and Immunity, 16, 74-84. doi 10.1006/brbi.2000.0619

Schlotz, W., Hammerfald, K., Ehlert, U., \& Gaab, J. (2011). Individual differences in the cortisol response to stress in young healthy men: Testing the roles of perceived stress reactivity and threat appraisal using multiphase latent growth curve modeling. Biological Psychology, 87 257-264. doi: 10.1016/j.biopsycho.2011.03.005
Schulz, P., Schlotz, W., \& Becker, P. (2004). Trierer Inventar zum chronischen Stress [Trier Inventory for the Assessment of Chronic Stress]. Göttingen, Germany: Hogrefe.

Smedes, F., Kraak, J. C., \& Poppe, H. (1982). Simple and fast solvent extraction system for selective and quantitative isolation of adrenaline, noradrenaline and dopamine from plasma and urine. Journal of Chromatography, 231, 25-39.

Sroussi, H. Y., Williams, R. L., Zhang, Q. L., Villines, D., \& Marucha, P. T. (2009). Ala42S100A8 ameliorates psychological-stress impaired cutaneous wound healing. Brain, Behavior, and Immunity, 23, 755-759. doi: 10.1016/j.bbi.2009.03.006

Stroncek, J. D., \& Reichert, W. M. (2008). Overview of wound healing in different tissue types. In W. M. Reichert (Ed.), Indwelling neural implants: Strategies for contending with the in vivo environment ( $\mathrm{pp}$. 3-40). Boca Raton, FL: CRC Press.

Subramaniam, M., Saffaripour, S., Van De Water, L., Frenette, P. S., Mayadas, T. N., Hynes, R. O., \& Wagner, D. D. (1997). Role of endothelial selectins in wound repair. American Journal of Pathology, 150, 1701-1709.

Tymen, S. D., Rojas, I. G., Zhou, X., Fang, Z. J., Zhao, Y., \& Marucha, P. T. (2013). Restraint stress alters neutrophil and macrophage phenotypes during wound healing. Brain, Behavior, and Immunity, 28, 207-217. doi: 10.1016/j.bbi.2012.07.013

Walburn, J., Vedhara, K., Hankins, M., Rixon, L., \& Weinman, J. (2009). Psychological stress and wound healing in humans: A systematic review and meta-analysis. Journal of Psychosomatic Research, 67, 253-271. doi: 10.1016/j.jpsychores.2009.04.002

Williams, R. L., Sroussi, H. Y., Abercrombie, J. J., Leung, K., \& Marucha, P. T. (2012). Synthetic decapeptide reduces bacterial load and accelerates healing in the wounds of restraint-stressed mice. Brain, Behavior and Immunity, 26, 588-596. doi: 10.1016/j.bbi.2012.01.020

Wirtz, P. H., Ehlert, U., Emini, L., \& Suter, T. (2008). Higher body mass index (BMI) is associated with reduced glucocorticoid inhibition of inflammatory cytokine production following acute psychosocial stress in men. Psychoneuroendocrinology, 33, 1102-1110. doi: 10.1016/ j.psyneuen.2008.05.002

Wirtz, P. H., Elsenbruch, S., Emini, L., Rudisuli, K., Groessbauer, S., \& Ehlert, U. (2007). Perfectionism and the cortisol response to psychosocial stress in men. Psychosomatic Medicine, 69, 249-255. doi: 10.1097/ PSY.0b013e 318042589e

Wirtz, P. H., von Kanel, R., Emini, L., Suter, T., Fontana, A., \& Ehlert, U. (2007). Variations in anticipatory cognitive stress appraisal and differential proinflammatory cytokine expression in response to acute stress. Brain, Behavior, and Immunity, 21, 851-859. doi: 10.1016/j.bbi .2007.02.003 